\title{
RESEARCH
}

Open Access

\section{Role of miR-146a in neural stem cell differentiation and neural lineage determination: relevance for neurodevelopmental disorders}

Lam Son Nguyen ${ }^{1,2^{*}}$, Julien Fregeac ${ }^{1,2}$, Christine Bole-Feysot ${ }^{1}$, Nicolas Cagnard ${ }^{1}$, Anand lyer ${ }^{3}$, Jasper Anink ${ }^{3}$, Eleonora Aronica ${ }^{3}$, Olivier Alibeu ${ }^{1}$, Patrick Nitschke ${ }^{1}$ and Laurence Colleaux ${ }^{1,2}$ (i)

\begin{abstract}
Background: MicroRNAs (miRNAs) are small, non-coding RNAs that regulate gene expression at the posttranscriptional level. miRNAs have emerged as important modulators of brain development and neuronal function and are implicated in several neurological diseases. Previous studies found miR-146a upregulation is the most common miRNA deregulation event in neurodevelopmental disorders such as autism spectrum disorder (ASD), epilepsy, and intellectual disability (ID). Yet, how miR-146a upregulation affects the developing fetal brain remains unclear.

Methods: We analyzed the expression of miR-146a in the temporal lobe of ASD children using Taqman assay. To assess the role of miR-146a in early brain development, we generated and characterized stably induced H9 human neural stem cell (H9 hNSC) overexpressing miR-146a using various cell and molecular biology techniques.

Results: We first showed that miR-146a upregulation occurs early during childhood in the ASD brain. In H9 hNSC, miR-146a overexpression enhances neurite outgrowth and branching and favors differentiation into neuronal like cells. Expression analyses revealed that $10 \%$ of the transcriptome was deregulated and organized into two modules critical for cell cycle control and neuronal differentiation. Twenty known or predicted targets of miR-146a were significantly deregulated in the modules, acting as potential drivers. The two modules also display distinct transcription profiles during human brain development, affecting regions relevant for ASD including the neocortex, amygdala, and hippocampus. Cell type analyses indicate markers for pyramidal, and interneurons are highly enriched in the deregulated gene list. Up to 40\% of known markers of newly defined neuronal lineages were deregulated, suggesting that miR-146a could participate also in the acquisition of neuronal identities.

Conclusion: Our results demonstrate the dynamic roles of miR-146a in early neuronal development and provide new insight into the molecular events that link miR-146a overexpression to impaired neurodevelopment. This, in turn, may yield new therapeutic targets and strategies.
\end{abstract}

Keywords: Autism spectrum disorders, microRNA, Human neural stem cell, Transcriptome

\footnotetext{
* Correspondence: lamson.nguyen@inserm.fr; lam-son.nguyen@inserm.fr 'INSERM UMR 1163, Laboratory of Molecular and pathophysiological bases of cognitive disorders, Imagine Institute, Necker-Enfants Malades Hospital, 24 Boulevard du Montparnasse, 75015 Paris, France

${ }^{2}$ Paris Descartes-Sorbonne Paris Cité University, 12 Rue de l'École de

Médecine, 75006 Paris, France

Full list of author information is available at the end of the article
}

(c) The Author(s). 2018 Open Access This article is distributed under the terms of the Creative Commons Attribution 4.0 International License (http://creativecommons.org/licenses/by/4.0/), which permits unrestricted use, distribution, and reproduction in any medium, provided you give appropriate credit to the original author(s) and the source, provide a link to the Creative Commons license, and indicate if changes were made. The Creative Commons Public Domain Dedication waiver (http://creativecommons.org/publicdomain/zero/1.0/) applies to the data made available in this article, unless otherwise stated. 


\section{Background}

Studies now indicate that epigenetic modifications play a role in neurodevelopmental disorders. The heritability rate of autism spectrum disorder (ASD) is over $50 \%$ with the remaining attributed to environmental/epigenetic factors [1]. MicroRNA (miRNA), one such factor, fine-tunes gene expression required for the development and function of cells and organs. Previously, our group and others implicated upregulation of $m i R-146 a$ as the most common miRNA deregulation event in ASD $[2,3]$ and related neurodevelopmental disorders such as epilepsy [4] and intellectual disability (ID) [2]. In ASD, studies reported miR-146a upregulation in olfactory mucosal stem cells [2], skin fibroblasts [2], and a lymphoblastoid cell line [5] sampled from living patients and the frontal cortex of adult post-mortem brain samples [6]. In post mortem samples from ASD brains [7], miR-146a promoter correlates with an increased level of the active H3K27ac histone mark suggesting that the observed upregulation is due to transcriptional deregulation. In epilepsy, $m i R-146 a$ is upregulated in astrocytes in region proximal to the lesions $[4,8]$. Importantly, treatment with either an anti-miR-146a [9] or a miR-146a mimic [10] can ameliorate the latency, frequency, and duration of induced seizures in a rat model of temporal lobe epilepsy, emphasizing the causality and the reversibility of $m i R-146 a$ effects. Understanding the functions of this miRNA in the brain may thus offer opportunities to develop treatments that are currently not available for neurodevelopmental disorders.

$m i R-146 a$ is independently transcribed and processed and evolutionary conserved to lower vertebrates such as zebrafish and fruit fly. In the mouse brain, it is expressed ubiquitously during embryonic development [2]. In postnatal stages, its expression becomes restricted to neurons in regions important for higher cognitive and social functions including frontal cortex, amygdala, and hippocampus [2]. $m i R-146 a$ is well known as a suppressor of inflammatory response by targeting TRAF6 and IRAK1 [11]. Its role in brain development is less well explored. In vitro data demonstrate that miR-146a regulates the homeostasis and function of brain cells in a developmental stage and cell type-specific manner. In primary mouse neural stem cell (NSC) cultured in EGF and FGF2, miR-146a overexpression promoted neuronal differentiation and cell cycle exit by targeting Notch1 [12]. In mature primary mouse neurons, its overexpression altered dendritic arborization [2] and induced AMPA receptor endocytosis [13], while transfection with the anti-miR-146a reduced the frequency and amplitude of synaptic transmission [13]. In rat primary NSC cultured in N2 and bFGF, overexpression of $m i R-146 a$ promoted astrocyte differentiation by inhibiting Syt1 and Nlgn1 expression [14]. In primary mouse astrocyte culture,
miR-146a overexpression hindered migration [12] and proliferation rate and increased glutamate uptake capacity [2].

Collectively, these studies suggest that $m i R-146 a$ contributes to the maintenance and differentiation of NSC. Yet, how miR-146a controls the equilibrium among key genes that promote or inhibit entry into the neurogenic program in human NSC remains unclear. We also do not know how this extrapolates to human brain development. To address these issues, we combined expression analyses in human brain samples and in vitro studies on H9-derived human NSC (H9 hNSC) modified to overexpress $m i R-146 a$.

\section{Methods}

\section{Patient information}

Freshly frozen brain samples were acquired from the NIH Neurobiobank from donors with ASD diagnosis and normal controls. There was no difference in the distribution of sex, ethnicity, average age, nor post-mortem interval (PMI) between the ASD and the control groups (results not shown). The causes of death vary; more ASD donors died from accidents, and more control donors died from infection and heart failure (see Additional file 1: Table S1).

\section{Whole exome sequencing technique and analysis}

DNA was extracted from frozen brain samples using QIAamp DNA Mini Kit (51304, QIAGEN) with RNAse A treatment (19101, QIAGEN) following the manufacturer's instruction. WES libraries were prepared from $3 \mu \mathrm{g}$ of genomic DNA sheared with a Covaris S2 Ultrasonicator. Exome capture was performed as recommended by the manufacturer with the $51 \mathrm{Mb}$ SureSelect Human All Exon kit V5 (Agilent technologies). Sequencing of the WES libraries was carried on a pool of barcoded exome libraries on a HiSeq2500 (Illumina) using the HighOutput mode (48 WES libraries per FlowCell). $76+76$ paired-end reads were generated. After demultiplexing, paired-end sequences were mapped on the human genome reference (NCBI build37/hg19 version) using BWA. The depth of coverage obtained for each sample was $>80 \times$ with $>90 \%$ of the exome covered at least by $15 \times$. SNPs and indels calling were made using GATK tools. An in-house software (PolyWeb) was used to annotate and filter the variants. Variant filtering was performed as previously described. Following a published protocol [2], we performed whole exome sequencing (WES) to identify possible deleterious single nucleotide variants (SNVs) in known ASD genes (as collated in SFARI Database) and intellectual disability genes (Necker ID-Panel) in patients (see Additional file 1: Table S2). SNVs in two patients (5308 and 4721) are known single nucleotide polymorphism (SNPs) and 
unlikely to be pathogenic. We could not confidently establish a genetic cause for most patients, except one. Patient 1349 carried a heterozygous SNV leading to premature termination codon in SEMA5A (OMIM 209850), which could explain his phenotype.

\section{Extraction and analysis of miRNAs}

Approximately $50 \mathrm{mg}$ of frozen brain samples were lysed in $600 \mu \mathrm{l}$ of Lysis/Binding solution from the miRVana ${ }^{\mathrm{mm}}$ miRNA isolation kit (AM1560, ThermoFisher Scientific) using FastPrep Lysing Matrix D (116913500, MP Biomedicals) on a FastPrep-24 Instrument (MP Biomedicals). The samples were spinned for $30 \mathrm{~s}$ at 13,000 RPM to reduce bubbles, after which extraction was performed according to the instruction of the manufacturer to obtain total RNA. The concentration was checked by NanoDrop 2000 (ThermoFisher Scientific). Expression profiles of miR-146a and 2 housekeeping miRNAs (miR-106a and miR-17) were assessed using Taqman assays on Fluidigm 98.98 array in technical quadruplicates. The qPCR analysis was carried out on the qPCR-HD-GPC core facility of the ENS and was supported by grants from Région Ile de France. The analysis was performed using mean of housekeeping miRNAs and average of all controls as references.

\section{Human neural stem cell culture and differentiation}

GIBCO $^{\circ}$ hNSC (H9 hESC-Derived) was purchased commercially (N7800100, ThermoFisher Scientific). Cells were cultured in flasks or plates previously coated for at least $1 \mathrm{H}$ with GelTrex ${ }^{\mathrm{Tn}}$ LDEV-Free, hESC-qualified, reduced growth factor basement membrane matrix (A1413302, ThermoFisher Scientific). Cells were maintained in Complete StemPro ${ }^{\circ}$ NSC SFM medium (growth media) consisting of KnockOut ${ }^{\text {tm }}$ D-MEM/F-12 (12660012, ThermoFisher Scientific) supplemented with 2\% StemPro ${ }^{\circ}$ Neural Supplement (A1050901, ThermoFisher Scientific), $20 \mathrm{ng} / \mathrm{mL}$ EGF (PHG0315, ThermoFisher Scientific), $20 \mathrm{ng} / \mathrm{mL}$ bFGF (GF003, ThermoFisher Scientific), $2 \mathrm{mM} \quad$ GlutaMax $^{\mathrm{mm}}-\mathrm{I}$ (35050038, ThermoFisher Scientific), and $10 \mu \mathrm{g} / \mathrm{mL}$ Penicillin-Streptomycin $(15,140,122$, ThermoFisher Scientific). For passaging, cells were washed once with DPBS (14190169, ThermoFisher Scientific) and detached from the surface by StemPro ${ }^{\text {Tx }}$ Accutase (A1110501, ThermoFisher Scientific), centrifuged at 1500 RPM for $5 \mathrm{~min}$ and replated in fresh growth media. To initiate spontaneous differentiation, H9 cells were plated at a density of 20,000 cells $/ \mathrm{cm}^{2}$ in growth media for $24 \mathrm{~h}$, after which media was replaced with differentiating media (growth media without growth factors). Differentiating media was changed every 2-3 days during the course of the differentiation process.

\section{Infection and selection of stably integrated $\mathrm{H} 9$ cells}

The seed of the miR-146a-5p in the pLenti-III-miR-146a-GFP construct (mm10082, ABM) 5'-T(GAGAACTG)AATTCCATGGGTT-3' was destroyed using the QuikChange site-directed mutagenesis kit (200515, Agilent) to produce to miR-146a-Mut construct $\quad 5^{\prime}$-T(_ATAGGAG)AATTCCATGGGTT-3' . Lentivirus were then produced from the construct by the Plateforme Vecterus Viraux et Transfert de Gènes from Hospital Necker as a paid service. Viral titer was determined by FACS of GFP signal. Virus infection was performed at the multiplicity of infection (MOI) of 5 in growth media, which was replaced after $24 \mathrm{~h}$. Cells were grown in growth media for 72 h; Puromycin (11113802, ThermoFisher Scientific) at $1 \mu \mathrm{g} / \mathrm{ml}$ was then added to the media for $72 \mathrm{~h}$ to eliminate all cells without stable integration of the viral DNA containing the transgene and the Puromycin resistance gene. Cells were recovered in normal growth media until reaching confluence. All experiments were performed at the earliest passages possible.

\section{Proliferation and apoptosis rate analysis}

Cells were plated on coated 96-well plate (92096, TPP) in normal growth media. Twenty-four hours after, media was replaced with growth media containing 1/200 Annexin V Incucyte reagent (4641, Essen Bioscience). Cells were cultured inside the Incucyte Live Cell Analysis System (Essen Bioscience), and images were taken at determined interval consecutively during several days using $\times 20$ objective and at multiple spots per well. In post-analysis, a mask was designed using an inbuilt apoptosis analysis module to count the red apoptotic cells and the cell confluence percentage, which was used to normalize for cell numbers (see Additional file 2: Figure S2a). The same mask was applied to all time points and all repeats.

\section{Differentiation analysis}

Cells were plated on coated 24-well plate (92024, TPP) in normal growth media. Twenty-four hours after, growth media was replaced with differentiating media, which was refreshed every 2-3 days. Cells were cultured inside the IncuCyte ${ }^{\circ}$ Live Cell Analysis System (Essen Bioscience), and images were taken every $3 \mathrm{~h}$ for 14 days using $\times 20$ objective and at 25 different spots per well. In post analysis, a mask was designed using the inbuilt neurite analysis module to detect neurites and cell body. The same mask was applied to all time points and all repeats to record neurite extension over time.

\section{FACS analysis}

Cells were plated on coated T25 tissue flask (90025, TPP) in normal growth media. Twenty-four hours after, growth media was replaced with differentiating media, 
which was refreshed every 2-3 days. Cells were collected at 1 and 2 weeks into the differentiation process by scraping the surface of the vessels. Cells were then washed once with DPBS and incubated on ice for $30 \mathrm{~min}$ with the blue fluorescent reactive dye from the Live/dead fixable dead cell stain kit (L23105, ThermoFisher Scientific). Cells were washed once with DPBS and fixed in $1 \mathrm{ml}$ of IC Fixation Buffer (FB001, ThermoFisher Scientific) for 5 min followed by 2 washes in IC Permeabilization Buffer (PB001, ThermoFisher Scientific) $+1 \%$ BSA. Cells were resuspended into $100 \mu \mathrm{l}$ of IC Permeabilization Buffer $+1 \%$ BSA and primary antibodies at the desired concentrations for $1 \mathrm{~h}$ on ice. Cells were washed twice in IC Permeabilization Buffer $+1 \%$ BSA and incubated in the same media with the secondary antibodies at desired concentrations for $1 \mathrm{~h}$ on ice. Cells were washed twice with DPBS $+1 \%$ BSA and analyzed immediately on the BD FACS Aria II machine (BD Biosciences). Primary antibody list: polyclonal rabbit anti-NESTIN (N5413, Sigma), monoclonal mouse anti-TUB-III eFluor 570 conjugated (41-4510, eBioscience), monoclonal mouse anti-GFAP eFluor $^{\circ} 660$ (50-9892, eBioscience); secondary antibody list: donkey anti-rabbit Alexa Fluor 555 (A31572, ThermoFisher Scientific).

\section{RNA sequencing technique and analysis}

We tested cells from three independent experiments using three consecutive passages. Total RNA was extracted using TRizol reagent (15596026, ThermoFisher Scientific) and RNeasy Mini Kit (74104, QIAGEN) with DNase I treatment step (79254, QIAGEN) following the manufacturer's protocol. The integrity of RNA was determined by RNA ScreenTape (5067-5576, Agilent Technologies) on the Agilent 4200 TapeStation (Agilent Technologies). RNA-seq libraries were prepared starting from $600 \mathrm{ng}$ of total RNA using the TruSeq Stranded mRNA LT Sample Prep Kit (Illumina) as recommended by the manufacturer. Half of the oriented cDNA produced from the poly-A+ fraction were PCR amplified (11 cycles). RNA-seq libraries were sequenced on an Illumina HiSeq2500 (Paired-End sequencing $130 \times 130$ bases, High Throughput Mode). A minimum of 10 million of paired-end reads was produced per library sample. Sequence reads were aligned to the human HG19 reference genome using the Burrows-Wheeler Alignment version 0.6.2.13. Raw and processed data for all samples are available for download from Gene Expression Omnibus (https://www.ncbi.nlm.nih.gov/geo/) under accession number GSE100670. RNA-Seq data were analyzed using a combination of three different tools namely limma [15], DESeq2 [16], and edgeR [17].
Quantitative reverse transcription PCR analysis (RT-qPCR) To validate the RNA-Seq results, we selected 27 genes with relevant neuronal function or corresponding to known miR-146a targets and tested their expression by quantitative RT-PCR (RT-qPCR) on the Fluidigm 48.48 chip. As six of these genes were identified as DEGs in both conditions, this analysis included 33 comparisons. The qPCR analysis was performed at the qPCR-HD-GPC core facility of the ENS and was supported by grants from Région Ile de France. The analysis was performed using geometric mean of four housekeeping genes (CYC1, GPBP1, RPL13A, and SDHA) and average of the miR-146a-Mut repeats as references. No significant difference was detected for the 4 housekeeping genes. Lists of primers can be found in Additional file 1: Table S5.

\section{In silico analyses}

Known targets of miR-146a were extracted from miRTarBase [18]. Predicted targets were extracted from miRDIP [19]; only genes predicted by at least three different programs were considered. Target prediction of miR-146a-mut was performed using miRDB [20]. Pathway and function enrichment was analyzed using Ingenuity Pathway Analysis (QIAGEN). Protein-protein interaction map was extracted from STRING database [21] and further analyzed using Cytoscape V3.4.0 (http:// www.cytoscape.org). Protein interaction enrichment was identified by ClusterOne Plugin downloaded from Cytoscape database. Analyses of expression in different brain regions throughout lifespan and brain cell type enrichment were performed using published code [22] in $\mathrm{R}$ program; please refer to the original publication for detailed description. Graphs and statistical analyses were generated by Partek Genomics Suite V6.0.

\section{Dual luciferase assay}

The 3' untranslated region (UTR) of $D C X, G A D 1$, and $P A K 3$ were subcloned into the psiCheck2 plasmid (Promega). Due to their size, the 3'UTR of DCX and PAK3 were cloned into 2 (924 and $810 \mathrm{bp)}$ and 3 parts (1106, 673 , and $524 \mathrm{bp}$ ), respectively; each of these parts contains at least 3 predicted miR-146a binding sites. For luciferase assay, $2 \times 10^{5}$ HEK293T cells were plated in each well of a 6-well plate the day before transfection. Cells were transfected with the psiCheck2 3'UTR clones and the synthetic miR-146a (4464067, ThermoFisher Scientific) or miR-mimic for control (4464058, ThermoFisher Scientific) using JetPRIME ${ }^{\odot}$ Polyplus Transfection Reagent (Ozyme) following the manufacturer's instruction. Assay were performed using the Dual-Luciferase ${ }^{\circ}$ Reporter Assay System (Promega) $48 \mathrm{~h}$ after transfection. Ratio of Renilla to Firefly luciferase was taken as mean 
of technical triplicates. Three independent transfections were performed to confirm the results.

\section{Results \\ miR-146a overexpression occurs early during human brain development}

ASD, which is diagnosed before the age of 3 , is associated with brain defects arising during early development, including enlarged brain volume (2-5\%) that ameliorates in later stages [23] and disorganized cortical layers [24]. As a first step to understand how miR-146a overexpression may contribute to ASD, we asked whether it could be detected early during brain development. Using a Taqman assay, we analyzed the expression of miR-146a and two housekeeping miRNAs (miR-106a and miR-17) in the temporal lobe (Brodmann's Area 21) of ASD children (4-9 years old) (see the "Methods" section and Additional file 1: Tables S1, S2 for detailed sample description). We observed a 1.3-fold increase in miR-146a expression in the patient samples compared to controls (see Fig. 1a), demonstrating that miR-146a upregulation is observed during the early stages of ASD progression/ emergence.

\section{Generation and validation $\mathrm{H} 9 \mathrm{hNSC}$ lines overexpressing miR-146a}

To elucidate the role of miR-146a in neural development, we used integrating viral approach to establish two H9 hNSC lines stably overexpressing a wild-type miR-146a gene or its mutant form, miR-146a-Mut (see Fig. 1b). Transgene expression was validated by either Taqman assay for miR-146a (see Fig. 1c) or measurement of the GFP transcript level by RT-qPCR for the miR-146a-Mut cell (see Fig. 1d). These lines were also validated using RNA-Seq, which showed a significant downregulation of known miR-146a target transcripts [18], including ICAM1, FAS, IRAK2, CFH, CDKN1A, TLR4, L1CAM, and CARD10 (see Fig. 1e). Predicted targets of $m i R-146 a$ were more frequently downregulated compared to those of miR-146a-Mut (20 vs. 5 targets with fold change $>1.5$ and $P<0.05$ in undifferentiated cells; $P<0.01$ by Fisher's Exact Test), suggesting that off-target effect due to miR-146a-Mut overexpression was minimal. In addition, array comparative genomic hybridization analysis did not identify any copy number variation, indicating that the genomes of the newly established lines were stable (results not shown).

\section{miR-146a promotes hNSC differentiation}

We then assessed the effects of miR-146a overexpression using live cell imaging (see Additional file 2: Figure S1a). In normal growth conditions, overexpression of miR-146a had no effect on the proliferation rate or the apoptotic rate of the cells (see Additional file 2: Figures
S1b ,c). In contrast, upon induction of differentiation by withdrawal of EGF and bFGF growth factors, we observed a significant decrease in the proliferation rate (see Fig. 2a). The apoptotic rates of the miR-146a and miR-146a-Mut lines increased during cell differentiation, but there was no difference between them (see Fig. 2b). Importantly, over the 2-week course of differentiation, we observed increased dendritic branching (see Fig. 2c) and extension (see Fig. 2d) in the miR-146a line compared to control. Undifferentiated H9 hNSC co-express NESTIN, $\beta$-III-TUBULIN (TUB-III), and GFAP [25], which are markers for progenitor, differentiated neuron and astrocyte, respectively. As differentiation proceeded, some cells lost their NESTIN expression and increased TUB-III expression, while GFAP level remained unchanged (see Additional file 2: Figure S2a,b). We calculated the ratio of cells expressing high level/low level of TUB-III and the number of NESTIN positive/negative cells after differentiation. We found that miR-146a overexpression induces enhanced level of TUB-III and reduced levels of NESTIN (see Fig. 2e). Collectively, these results suggest that $m i R-146 a$ overexpression favors differentiation of $\mathrm{H} 9 \mathrm{hNSC}$ into neurons in response to neurogenic cue.

miR-146a overexpression alters the balance between neural progenitor cell renewal and neuronal differentiation miRNAs regulate multiple signaling pathways through their interaction with hundreds of different transcripts. To identify the mechanisms that could contribute to neural differentiation in miR-146a overexpressing hNSC, we performed expression analyses. Proteomic analysis is a valuable approach to identify changes in protein levels regulated by $m i R-146 a$, yet, it presents important limitations including limited access to low abundant proteins which expression is triggered during differentiation. Thus, we decided to perform RNA-Seq analyses in both undifferentiated and differentiated cells to identify differentially expressed genes (DEGs) and corresponding affected pathways that may mediate the effect of $m i R-146 a$ overexpression on neural differentiation. Compared to miR-146a-Mut using a threshold of $P<0.05$ and fold change $>1.5$, we detected 1185 DEGs $(10 \%$ of total detected transcripts, 54\% downregulated, and 46\% upregulated) in undifferentiated cells (see Fig. $3 \mathrm{a}$ and Additional file 1: Table S3) and 1039 DEGs (9\% of total detected transcripts, 59\% down, and 41\% upregulated) in differentiated cells (see Fig. 3a and Additional file 1: Table S4). We obtained a $97 \%$ validation rate by RT-qPCR (32/33 genes validated, $R^{2}=0.978$; see the "Methods" section; see Fig. $3 \mathrm{~b}$ and Additional file 1: Table S5). We further validated the downregulation of PAK3 in undifferentiated and differentiated H9 NSC, 


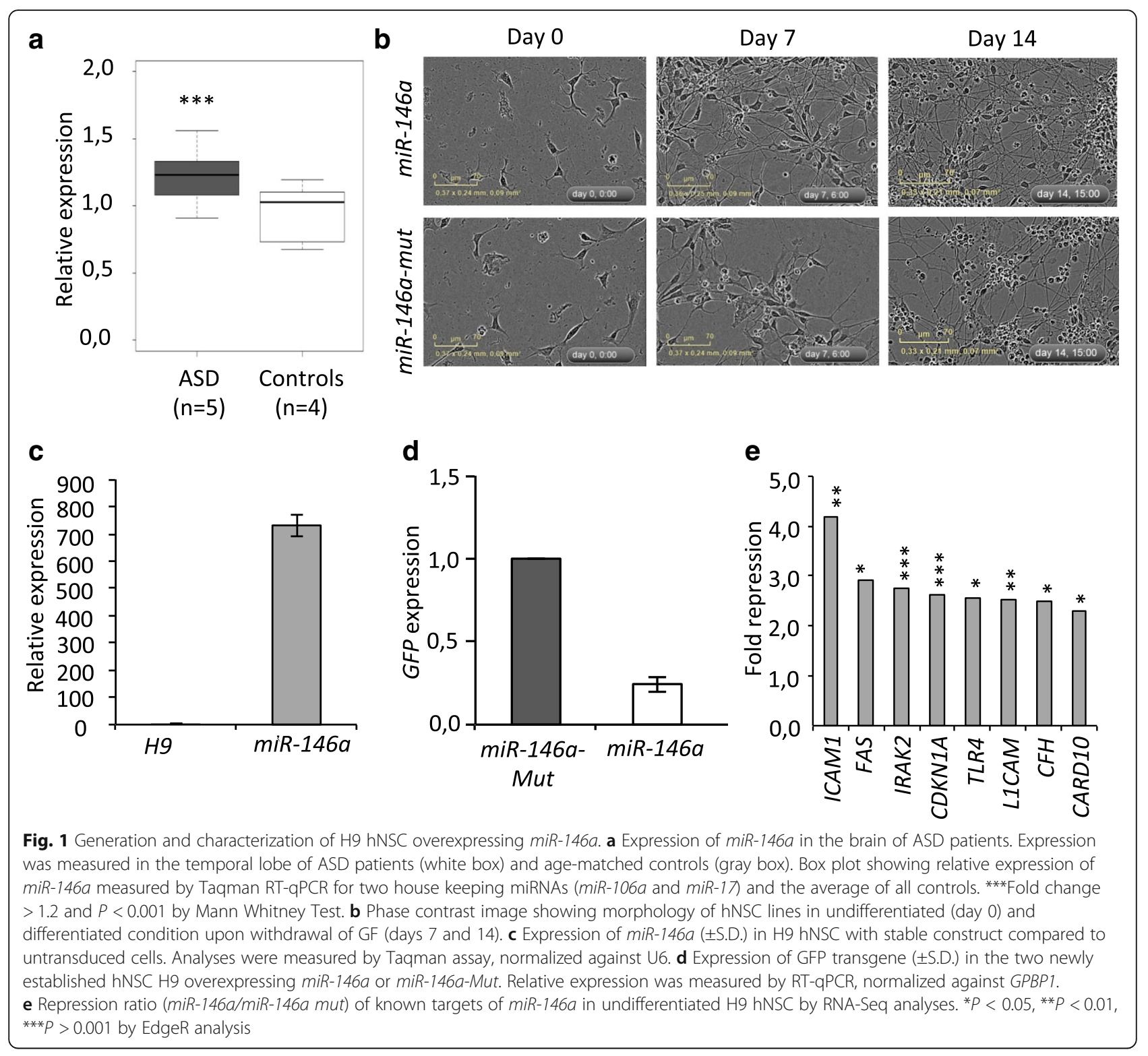

and the upregulation of NOTCH1 in differentiated cells by western blot (Additional file 2: Figure S3).

Next, we sought to identify the deregulated pathways. We first extracted high confident known and predicted interactions from STRING database [21]. In undifferentiated cells, pathway analyses suggested that DEGs affect very different classes of biological processes including axonal guidance, colorectal cancer, germ Cell-Sertoli Cell Junction Signaling (see Additional file 1: Table S6). Moreover, gene/protein interacting network was random and did not organize into functional modules (see Additional file 2: Figure S4). In contrast, analysis on data obtained from differentiated cells revealed enrichment for two networks (see Fig. 3c). Using Cytoscape with ClusterOne plugin, we found that the first network consisted of 155 genes comprising of four modules (see
Additional file 2: Figure S5 and Additional file 1: Table S7). Remarkably, all genes from this network except one are downregulated (154/155 DEGs, $P=0$ by Fisher's Exact Test) and were enriched for cell cycle control pathways (Cell Cycle Module; see Fig. 3d and Additional file 1: Table S8). We further analyzed the remaining 884 genes by filtering out those encoding proteins with no or only one interaction (those in the outer edge). This created a second list of 350 genes with an equal distribution of up or downregulated DEGs (195 down, 153 upregulated, $P>0.05$ by Fisher's Exact Test). Importantly, a significant enrichment for pathways related to neuronal differentiation was observed in this dataset (Neuronal Module; see Fig. 3c and Additional file 1: Table S9). To identify the drivers that could mobilize the observed interactions, we search for known and predicted miR-146a 

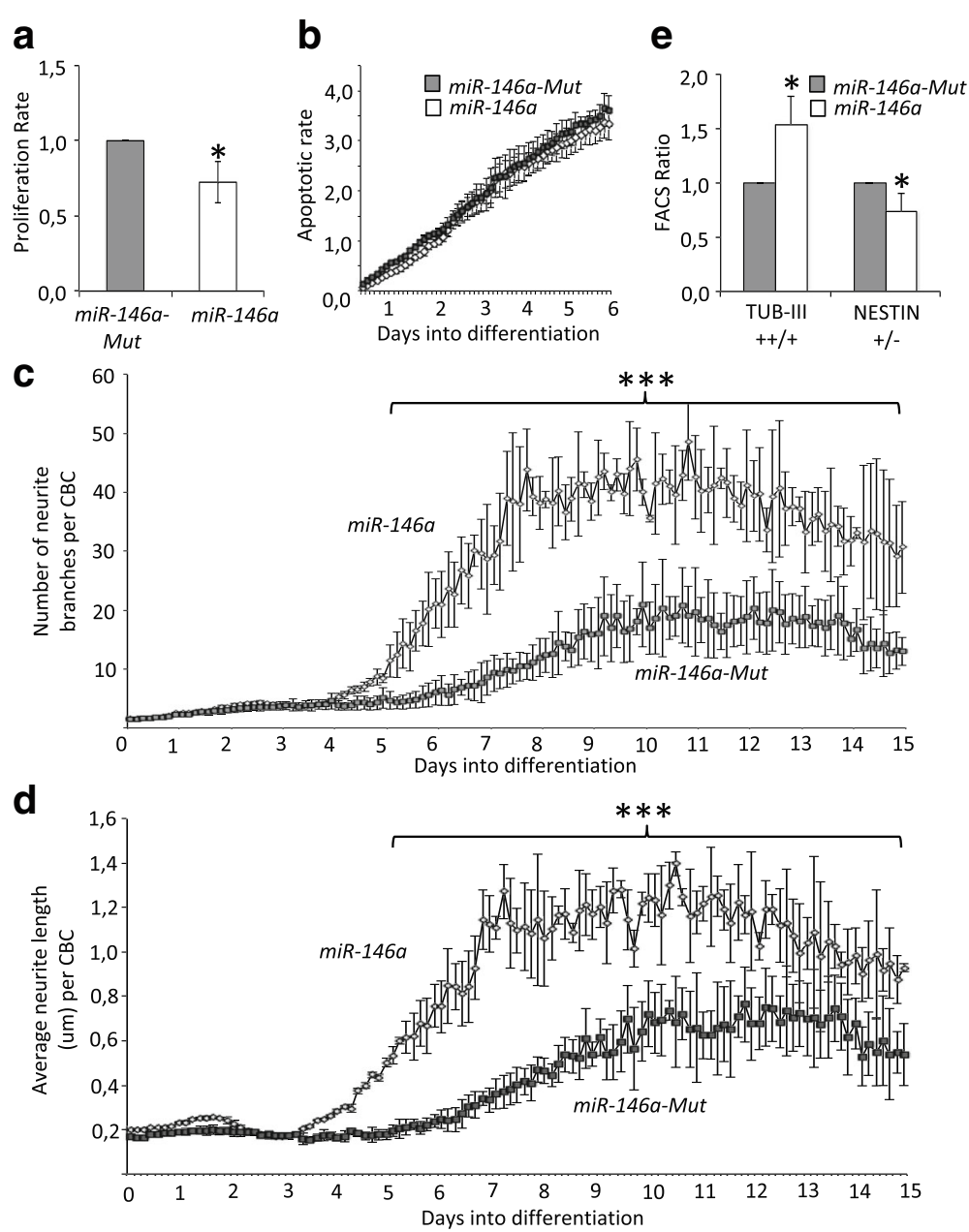

Fig. 2 miR-146a controls responses of H9 hNSC to neurogenic cues. a Cell proliferation rates measured using the Incucyte machine over $48 \mathrm{~h}$ after induction of differentiation. Graph shows the average ratio ( \pm S.D.) of proliferation slopes from 3 independent experiments. ${ }^{*} P<0.05$ by Student's 2-tail unpaired $T$ test. b Apoptotic cell rates measured by the number of Annexin $V$ labeled cells over the confluence percentage using the Incucyte machine. Graph shows average ratio ( \pm S.D.) of four technical replicates and are representative of two independent repeats showing the same results. $\mathbf{c}$ Average number of neurite branching ( \pm S.D.) and average neurite length $\mathbf{d}( \pm S . D$.) in cells undergoing differentiation over 2 weeks. Analyses were performed using an analysis mask (see Additional file 2: Fig. S1a) on images taken at 25 different spots every $3 \mathrm{~h}$ over 15 days by the Incucyte machine. Results are representative of three independent repeats showing similar results. ${ }^{* *} P<0.001$ by Student's 2-tailed unpaired $T$ test. e FACS analysis was performed on differentiated cells labeled by TUB-III or NESTIN antibodies (see Additional file 2: Figure S2 for details). Graph shows average ratio ( \pm S.D.) of three independent repeats. ${ }^{*} P<0.05$ by Student's 2 -tail unpaired $T$ test

targets (as collated in miRTarBase [18] and using at least 3 different programs as collated by miRDiP [19]) that co-localized to this network (see Additional file 2: Figure S6). We chose to test the interaction between miR-146a and $P A K 3, D C X$ and $G A D 1$ by luciferase assays for their crucial roles in NSC differentiation and neuronal migration. This analysis revealed that miR-146a directly targets the 3'UTR of all three genes (see Fig. 3e). Taken together, our results suggested that miR-146a promotes cell cycle exit and neuronal differentiation by targeting 11 key cell cycle genes (CHEK1, BRCA1, BRCA2, CCNA2, CDKN1A, CDKN3, TIMELESS, CDK1, CDCA5, $E 2 F 2 \& K I F 18 A)$ and 9 neuronal genes (DCX, PAK3,
IRS1, GAD1, SLC17A8, EPB41, MYBL1, IQGAP3 \& $L I N 28 B), 9$ of which are validated targets while 11 are predicted targets of miR-146a (see Fig. 3f).

\section{miR-146a overexpression affects specific regions in early human brain development}

To identify the developmental stages and regions of the human brain potentially affected by the overexpression of miR-146a, we investigated the expression of the DEGs in a comprehensive dataset on the human brain transcriptome previously described $[22,26]$. We found that expression of genes in the Cell Cycle Module is specifically restricted to early stages of fetal development in the 
a

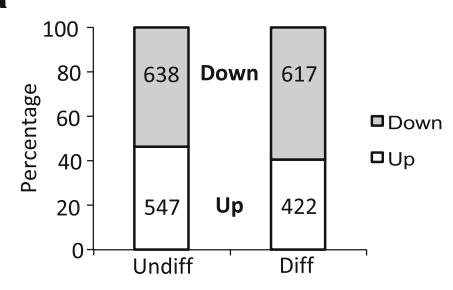

C

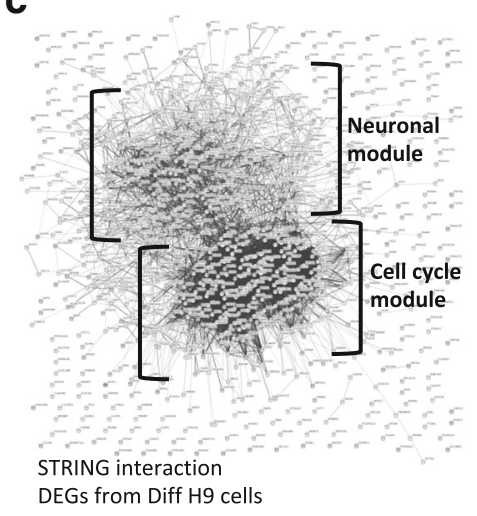

e

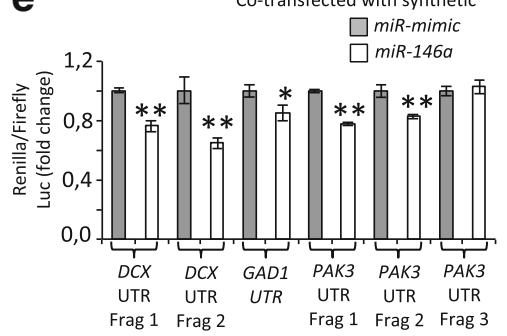

b

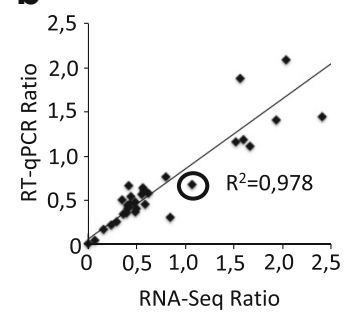

d
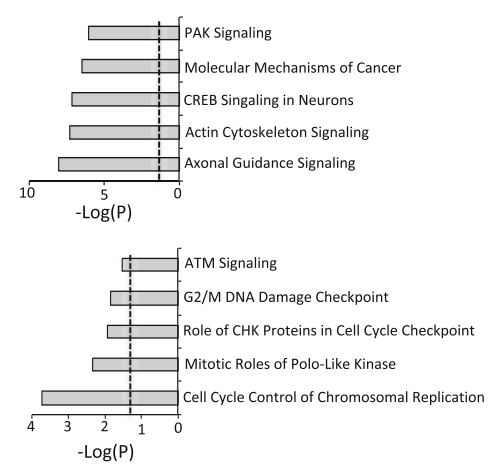

$f$

\begin{tabular}{|l|l|l|}
\hline Module & Predicted targets & Validated targets \\
\hline Neuronal & $\begin{array}{l}\text { IRS1, SLC17A8, } \\
\text { EPB41, IQGAP3, } \\
\\
\text { LIN28B, MYBL1 }\end{array}$ & DCX, GAD1, PAK3 \\
\hline Cell Cycle & TIMELESS, & CHEK1, BRCA1, \\
& CDCA5, E2F2, & BRCA2, CCNA2, \\
& CDK1, KIF18A & CDKN1A, CDKN3 \\
\hline
\end{tabular}

Fig. 3 miR-146a targets key genes to promote hNSC differentiation. a Distribution of DEGs identified by RNA-Seq in undifferentiated (Undiff) and differentiated (Diff) conditions. b Graph shows expression ratios of DEGs measured by RNA-Seq and RT-qPCR. Circle indicates only incompatible/ non-validated DEGs. c Protein interaction network of all DEGs identified in differentiated cells mapped by STRING. Two modules are identified: Neuronal Module (top) contains 350 genes and Cell Cycle Module (bottom) contains 155 genes. $\mathbf{d}$ Top 5 pathways identified by Ingenuity Pathway Analysis of the 2 modules (see Additional file 1: Tables S8, S9 for extended lists). e miR-146a directly targets DCX, GAD1, and PAK3 3'UTR. Due to their size, the $3^{\prime} U T R$ of DCX and PAK3 were cloned into 2 and 3 fragments (frag), respectively. Each $3^{\prime} U T R$ construct contains at least 3 predicted miR-146a binding sites. Ratio of Renilla/Firefly luciferase ( \pm SD of 3 technical triplicates) was measured in cell lines co-transfected with either the synthetic miR-mimic (control) or the miR-146a. Graphs are representative of 3 independent assays showing the same results. ${ }^{*} P<0.05$, ${ }^{* *} P<0.01$ by Student's 2 tailed paired $t$ test. $\mathbf{f}$ List of known and predicted miR-146a targets that are downregulated in each respective module

hippocampus, amygdala, visual cortex, medial prefrontal cortex, and cerebellum (see Fig. 4a). By contrast, we observed a developmental gradient of expression of genes from the Neuronal Module that increases during fetal development in the hippocampus and amygdala, peaks at an early infancy stage in all regions of the brain and persists throughout the life. Cerebellar expression seems restricted to prenatal stages (see Fig. 4b). Notably, this Neuronal Module, but not Cell Cycle Module, is significantly enriched for ASD-associated genes $(P<0.001$ by Fisher's Exact Test, see Additional file 1: Table S4).

To further explore the contribution of miR-146a upregulation on the transcriptome of ASD brain, we compared the DEGs identified in differentiated H9 hNSC to the published DEGs identified in ASD adult post-mortem brains (cortex, temporal lobe, and frontal cortex) [27]. We identified 42 overlapping genes that share the same trend of deregulation between the two studies (6.3\% of published DEGs detectable in H9 hNSC) (see Additional file 1: Table S4). Half of these genes (20/ 42) belong to the Neuronal Module, whereas only two belong to the Cell Cycle Module. This analysis suggested that upregulation of $m i R-146 a$ could be responsible for a portion of transcriptomic changes seen in ASD brains and that the use of $\mathrm{H} 9 \mathrm{hNSC}$ could provide relevant insights into both early processes of neural differentiation and later stages of neuronal development. 

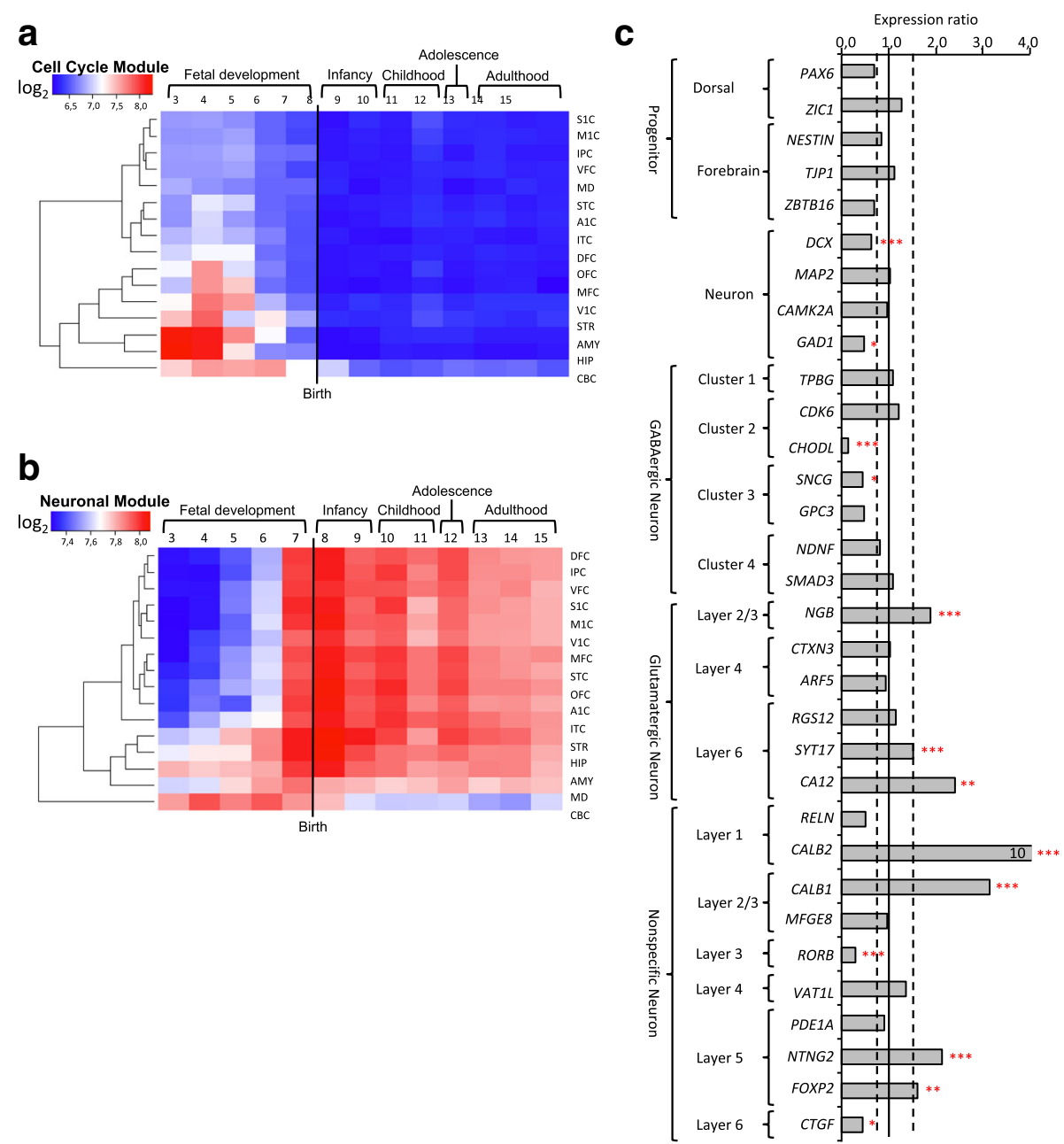

Fig. 4 miR-146a determines neuronal lineage identities. Heatmap of gradient of expression from the Cell Cycle (a) and Neuronal (b) Modules spanning human fetal development to late adulthood in distinct brain regions. A1C, auditory cortex; AMY, amygdala; CBC, cerebellar cortex; DFC, dorsolateral prefrontal cortex; HIP, hippocampus; IPC, posterior inferior parietal cortex; ITC, inferior temporal cortex; M1C, primary motor cortex; MD, mediodorsal nucleus of thalamus; MFC, medial prefrontal cortex; OFC, orbital prefrontal cortex; S1C, primary somatosensory cortex; STC, superior temporal cortex; STR, striatum; V1C, primary visual cortex; VFC, ventrolateral prefrontal cortex. Period labels: $3-$ Early fetal $(10 \leq$ Age $\leq 13$ Post conception week, PCW); 4 -Early midfetal ( $13 \leq$ Age $\leq 16$ PCW); 5 -Early midfetal ( $16 \leq$ Age $\leq 19$ PCW); 6 -Late midfetal $(19 \leq$ Age $\leq 24$ PCW); 7-Late fetal (24 $\leq$ Age $\leq 38$ PCW); 8 -Neonatal and early infancy (Birth $\leq$ Age $\leq 6$ Postnatal months, M); 9 -Late infancy $(6 \leq$ Age $\leq 12 \mathrm{M})$; 10-Early childhood ( $1 \leq$ Age $\leq 6$ Postnatal years, Y); 11 -Middle and late childhood ( $6 \leq$ Age $\leq 12$ Y); 12 —Adolescence $(12 \leq$ Age $\leq 20$ Y); 13-Young adulthood ( $20 \leq$ Age $\leq 40$ Y); 14 -Middle adulthood ( $40 \leq$ Age $\leq 60$ Y); 15 -Late adulthood (60 Y $\leq$ Age). c Expression difference of detectable neuronal specific markers in differentiated H9 hNSC. Dotted lines indicate expression difference threshold (fold change $\geq 1.5$ ).

${ }^{*} P<0.05,{ }^{*} P<0.01$ and ${ }^{* *} P<0.001$ by EdgeR analysis (as representative of 3 different RNA-Seq analyses)

\section{miR-146a overexpression correlates with abnormal lineage-specific gene expression}

The central nervous system contains many diverse neuronal subtypes that have been classified into 50 different groups based on single-cell gene expression profiles [28]. Since miR-146a overexpression alters hNSC differentiation, we asked whether it also affects neural cell fate programming and impacts neuronal specialization. Our RNA-seq data indicated that the Neuronal Module is enriched for genes expressed in pyramidal neurons and interneurons $(P<0.05$; see Additional file 1: Table S10) [22], even though the Cell Cycle Module is broadly expressed in all cell types (see Additional file 1: Table S10). When examining the expression of all 87 established cortical layer-specific, neuronal-specific, and progenitor-specific markers from published sources [24, 28, 29], we observed that $40 \%(13 / 32)$ of detectable markers for differentiated H9 cells were significantly deregulated in hNSC overexpressing miR-146a (see Fig. 4c). These markers were for GABAergic neurons (CHODL, GAD1, SNCG), glutamatergic neurons (NGB,SYT17, CA12), layers 2/3 (CALB1, $C A L B 2)$, layer $4(R O R B)$, layers 5/6 (NTNG2, FOXP2, $C T G F)$, and migrating neurons $(D C X)$ (see Fig. 4c). These results strongly suggest that regulation of $m i R-146 a$ 
expression level is important to correctly acquire neuronal lineage identities.

\section{Discussion}

In this study, we provided insight into the role of miR-146a in brain development and its relevance for neurodevelopmental disorders by combining the analyses of post-mortem human brain samples and in vitro models. We demonstrated that miR-146a overexpression in the brain of ASD patient is an early event detectable from childhood at an age when ASD are typically diagnosed (see Fig. 1a). Albeit the limited number of samples tested, this is the fifth time that this miRNA has been found upregulated in an independent cohort $[2,3,5,6]$. In neural-relevant cell types, upregulation of miR-146a has been reported in adult cortex BA10 [6] and in adult olfactory mucosal stem cells (OMSC) [2]. These results collectively suggest that miR-146a upregulation is an event that occurs during embryogenesis and continues throughout development.

Our in vitro analyses on hNSC suggest that miR-146a contributes to the regulation of balancing cell-cycle exit/ cell-cycle re-entry of neural progenitors and committing to neural differentiation pathways. These results are in agreement with previously published studies. Indeed, overexpression of $m i R-146 a$ has been shown to induce cell cycle arrest in normal [2] or malignant mouse astrocytes [12], in human non-small cell lung cancer cells [30] and in mouse NSC [12]. Increased miR-146a level also enhanced neuronal differentiation in mouse NSC through suppression of Notch1 [12]. However, in our study, we found that NOTCH1 was upregulated in differentiated H9 NSC. This can be explained by two major differences: (i) unlike the mouse Notch1, the human NOTCH1 gene is not predicted to be a target of $m i R-146 a$, and (ii) activation and not suppression of NOTCH1 is required for human NSC differentiation [31]. This emphasizes the relevance of using human H9 NSC to model early neuronal development of patients with ASD. In addition, we propose that miR-146a modulates the homeostasis of NSC by targeting directly and concurrently at least 20 different key neuronal and cell cycle genes, 9 of which are validated targets while 11 are predicted targets (see Fig. 3f). Thus, while inhibition of vesicular glutamate transporters (VGLUTs) promotes neuronal differentiation and migration of NPCs [32], we observed that miR-146a overexpression caused downregulation of the vesicular glutamate transporter gene SLC17A8. Similarly, RNA-Seq data revealed downregulation of the LIN28B gene; the RNA binding protein LIN28B plays essential functions in neuroblast proliferation by maintaining neural progenitors in an early state [33]. Lastly, miR-146a overexpression downregulates several CDK genes including CDKN1A, CDKN3, and $C D K 1$, which encode for key proteins controlling the length of $G_{1}$ phase during cell-cycle and the balance between progenitor maintenance and generation of differentiated neurons [34].

Cortical cytoarchitecture relies on the spatiotemporal coordination of neuronal production rate, precursors cell-cycle control, and neuronal radial migration toward the cortical plate. Radial glial cells (RGC), the key progenitor cells in the developing CNS, divide asymmetrically to generate a new RGC as well as a post-mitotic neuron or an intermediate progenitor daughter cell. Neurons migrate toward the cortical plate along the fibers of RGC to reach their final position within the nascent neocortex and acquire their specific identity. Migration and final laminar positioning of neurons relies the fine-tuning of cell type- and layer-specific transcription factors [35]. Our results suggest that upregulation of miR-146a could disturb these transcriptional programs and may contribute to the disorganization of cortical layers [24] and the increase in number of neurons [36] and dendritic spine density [37] observed in ASD brains.

Data from RNA-Seq indicate that DEGs in $\mathrm{H} 9$ hNSC are significantly enriched for markers for pyramidal and interneurons, as well as markers for GABAergic (up regulated) and glutamatergic neurons (down regulated) (see Fig. $4 \mathrm{c}$ and Additional file 1: Table S10), suggesting that miR-146a may also contribute to the adequate distribution of these neurons. In mouse, artificially enhanced number of pyramidal neurons in the upper neocortical layers impaired neurite extension and laminar distribution of interneurons, ultimately leading to autism-like phenotype [38]. In human, Wegiel et al. examined post-mortem brains of 14 subjects with ASD and reported localized deficit of pyramidal neurons in the CA1 sector in 3 subjects and thickening of pyramidal layer in the CA1 sector in another [39]. The number of PVALB positive interneurons, a GABAergic subtype, was also found significantly reduced in prefrontal cortex BA46, BA47, and BA9 in 11 ASD cases [40]. As such, our results will direct future investigations into the role of miR-146a in the signaling cascade mediating the determination and acquisition of neuronal lineage identities.

\section{Conclusion}

The accurate generation of an appropriate number of different neuronal and glial subtypes is fundamental for normal brain functions. It requires tightly orchestrated, spatial, and temporal developmental programs to maintain the balance between neural progenitor cell proliferation and differentiation. While ASD is often considered as caused by synaptic dysfunction [41], several evidence from human neuropathology, 
systems biology, and developmental biology implicated dysregulation of the cell cycle and cortical lamination in the developing brain as a potential common pathophysiological mechanism underlying ASD [42-45]. Based on our results, we speculate that miR-146a plays a dynamic role to shape brain development from early neurogenesis to synaptic maturation and propose that miR-146a overexpression could provide a potential unifying explanation for brain dysfunctions observed in neurodevelopmental disorders.

\section{Additional file}

Additional file 1: Table S1. Detailed information of all patients and controls whose brain samples were used in this study. Table S2. Possibly deleterious variants in known ASD and ID genes. Table S3. DEGs identified in undifferentiated cells. Table S4. DEGs identified in differentiated cells. Table S5. Validation of RNA-Seq using RT-qPCR on Fluidigm array. Table S6. Top 20 cannonical pathways deregulated in undifferentiated cells. Table S7. Top 4 nodes enriched for protein-protein interaction as calculated by ClusterOne Plugin. Table S8. Top 20 cannonical pathways deregulated in the cell cycle modules of differentiated cells. Table S9. Top 20 cannonical pathways deregulated in the cell neuronal modules of differentiated cells. Table S10. Cell type enrichment analysis of DEGs from the Cell Cycle and Neuronal Modules. (XLSX 888 kb)

Additional file 2: Figure S1. Characteristics of undifferentiated H9 hNSC. Figure S2. FACS analyses of cell type specific markers NESTIN, GFAP and TUB-III in undifferentiated and differentiated conditions. Figure S3. Western blot validation of PAK3 and NOTCH1 expression in undifferentiated and differentiated H9 NSC. Figure S4. Protein interaction network of all DEGs in undifferentiated cells predicted by STRING. Figure S5. Top four interacting networks corresponding to the cell cycle module in differentiated cells. Figure S6. Co-localization of known and predicted targets of miR-146a in the protein interaction network of DEGs in differentiated cells. (PPTX $7099 \mathrm{~kb}$ )

\section{Abbreviations}

ASD: Autism spectrum disorder; CNS: Central nervous system:

DEG: Differentially expressed gene; hNSC: Human neural stem cell; ID: Intellectual disability; miRNA: MicroRNA; MOI: Multiplicity of infection; OMSC: Adult olfactory mucosal stem cell; PMI: Post-mortem interval; RGC: Radial glial cell; RT-qPCR: Quantitative reverse transcription polymerase chain reaction; SNP: Single nucleotide polymorphism; SNV: Single nucleotide variant; TUB-III: $\beta$-III-TUBULIN; WES: Whole exome sequencing

\section{Acknowledgements}

Human tissues were obtained from University of Maryland Brain and Tissue Bank, which is a Brain and Tissue Repository of the NIH Neurobiobank. We would like to thank Bertrand Ducos, Juliette Pouch, and Elise Diaz for their help with the Fluidigm analysis. We would like to thank the core platforms of the Imagine Institute, notably the Cell Imagining Platform, the Gene Transfer Vector Platform for the virus production, the Cell Sorting Facility for their help with performing the FACS analyses and the Histology and Morphology Facility for their help with paraffin inclusion of brain samples and advice on analyses.

\section{Funding}

This work received a state subsidy managed by the National Research Agency under the "Investments for the Future" program bearing the reference ANR-10-IHU-01 and the "ANR-SAMENTA 2012" program. This work was also supported by the Fondation pour la Recherche Médicale (DEQ20160334938), the Fondation de France and the MSDAvenir Fund (Devo-Decode Project). LC is supported by the Centre National de la Recherche Scientifique. JF is supported by Crédit Agricole d'lle-de-France Mécénat. We thank Life Science Editors for editing assistance.

\section{Availability of data and materials}

The RNA-Seq data are available for download from Gene Expression Omnibus (https://www.ncbi.nlm.nih.gov/geo/) under accession number GSE100670.

\section{Authors' contributions}

LSN and LC designed and supervised the research. LSN performed all the experiments unless specified otherwise. JF cloned the miRNA expression constructs. CBF and OA performed the next generation sequencing experiments. $\mathrm{NC}$ and PN analyzed the next generation sequencing data. Al, JA, and EA helped analyze the human tissue biopsies. LSN and LC wrote the manuscript. All the authors reviewed the manuscript and approved the final manuscript.

\section{Ethics approval and consent to participate}

Brain biopsies were obtained from the University of Maryland Brain and Tissue Bank, which is a Brain and Tissue Repository of the NIH Neurobiobank. Consents were given to the Neurobiobank to redistribute biopsies for approved research (NBB_302).

\section{Competing interests}

The authors declare no competing interests.

\section{Publisher's Note}

Springer Nature remains neutral with regard to jurisdictional claims in published maps and institutional affiliations.

\section{Author details}

${ }^{1}$ INSERM UMR 1163, Laboratory of Molecular and pathophysiological bases of cognitive disorders, Imagine Institute, Necker-Enfants Malades Hospital, 24 Boulevard du Montparnasse, 75015 Paris, France. ${ }^{2}$ Paris Descartes-Sorbonne Paris Cité University, 12 Rue de l'École de Médecine, 75006 Paris, France. ${ }^{3}$ Department of (Neuro) Pathology, Academic Medical Center, University of Amsterdam, 1105 AZ Amsterdam, The Netherlands.

Received: 4 January 2018 Accepted: 30 May 2018

Published online: 19 June 2018

\section{References}

1. Sandin S, Lichtenstein P, Kuja-Halkola R, Larsson H, Hultman CM, Reichenberg A. The familial risk of autism. JAMA. 2014;311:1770-7.

2. Nguyen LS, Lepleux M, Makhlouf M, Martin C, Fregeac J, Siquier-Pernet K, Philippe A, Feron F, Gepner B, Rougeulle C, et al. Profiling olfactory stem cells from living patients identifies miRNAs relevant for autism pathophysiology. Mol Autism. 2016; https://doi.org/10.1186/s13229-0150064-6.

3. Fregeac J, Colleaux L, Nguyen LS. The emerging roles of MicroRNAs in autism spectrum disorders. Neurosci Biobehav Rev. 2016;71:729-38.

4. Iyer A, Zurolo E, Prabowo A, Fluiter K, Spliet WG, van Rijen PC, Gorter JA, Aronica E. MicroRNA-146a: a key regulator of astrocyte-mediated inflammatory response. PLoS One. 2012;7:e44789.

5. Talebizadeh Z, Butler MG, Theodoro MF. Feasibility and relevance of examining lymphoblastoid cell lines to study role of microRNAs in autism. Autism Res. 2008;1:240-50

6. Mor M, Nardone S, Sams DS, Elliott E. Hypomethylation of miR-142 promoter and upregulation of microRNAs that target the oxytocin receptor gene in the autism prefrontal cortex. Mol Autism. 2015;6:46.

7. Sun W, Poschmann J, Cruz-Herrera Del Rosario R, Parikshak NN, Hajan HS, Kumar V, Ramasamy R, Belgard TG, Elanggovan B, Wong CC, et al. Histone Acetylome-wide Association Study of Autism Spectrum Disorder. Cell. 2016; 167:1385-97. e1311

8. Aronica E, Fluiter K, lyer A, Zurolo E, Vreijling J, van Vliet EA, Baayen JC, Gorter JA. Expression pattern of miR-146a, an inflammation-associated microRNA, in experimental and human temporal lobe epilepsy. Eur J Neurosci. 2010;31:1100-7.

9. He F, Liu B, Meng Q, Sun Y, Wang W, Wang C. Modulation of miR-146a/ complement factor $\mathrm{H}$ mediated inflammatory responses in a rat model of temporal lobe epilepsy. Biosci Rep. 2016;36(6):e00433.

10. Tao H, Zhao J, Liu T, Cai Y, Zhou X, Xing H, Wang Y, Yin M, Zhong W, Liu Z, et al. Intranasal delivery of miR-146a mimics delayed seizure onset in the lithium-pilocarpine mouse model. Mediat Inflamm. 2017;2017:6512620. 
11. Taganov KD, Boldin MP, Chang KJ, Baltimore D. NF-kappaB-dependent induction of microRNA miR-146, an inhibitor targeted to signaling proteins of innate immune responses. Proc Natl Acad Sci U S A. 2006;103:12481-6.

12. Mei J, Bachoo R, Zhang CL. MicroRNA-146a inhibits glioma development by targeting Notch1. Mol Cell Biol. 2011;31:3584-92.

13. Chen $\mathrm{YL}$, Shen CK. Modulation of mGluR-dependent MAP1B translation and AMPA receptor endocytosis by microRNA miR-146a-5p. J Neurosci. 2013;33: 9013-20.

14. Jovicic A, Roshan R, Moisoi N, Pradervand S, Moser R, Pillai B, Luthi-Carter R. Comprehensive expression analyses of neural cell-type-specific miRNAs identify new determinants of the specification and maintenance of neuronal phenotypes. J Neurosci. 2013;33:5127-37.

15. Ritchie ME, Phipson B, Wu D, Hu Y, Law CW, Shi W, Smyth GK. Limma powers differential expression analyses for RNA-sequencing and microarray studies. Nucleic Acids Res. 2015:43:e47.

16. Love MI, Huber W, Anders S. Moderated estimation of fold change and dispersion for RNA-seq data with DESeq2. Genome Biol. 2014;15:550.

17. Robinson MD, McCarthy DJ, Smyth GK. edgeR: a Bioconductor package for differential expression analysis of digital gene expression data. Bioinformatics. 2010;26:139-40.

18. Chou CH, Chang NW, Shrestha S, Hsu SD, Lin YL, Lee WH, Yang CD, Hong HC, Wei TY, Tu SJ, et al. miRTarBase 2016: updates to the experimentally validated miRNA-target interactions database. Nucleic Acids Res. 2016;44: D239-47.

19. Shirdel EA, Xie W, Mak TW, Jurisica I. NAViGaTing the micronome-using multiple microRNA prediction databases to identify signalling pathwayassociated microRNAs. PLoS One. 2011;6:e17429.

20. Wong N, Wang X. miRDB: an online resource for microRNA target prediction and functional annotations. Nucleic Acids Res. 2015;43:D146-52.

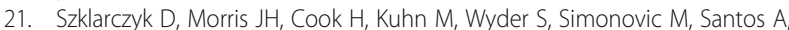
Doncheva NT, Roth A, Bork P, et al. The STRING database in 2017: qualitycontrolled protein-protein association networks, made broadly accessible. Nucleic Acids Res. 2017;45:D362-8.

22. Johnson MR, Shkura K, Langley SR, Delahaye-Duriez A, Srivastava P, Hill WD, Rackham OJ, Davies G, Harris SE, Moreno-Moral A, et al. Systems genetics identifies a convergent gene network for cognition and neurodevelopmental disease. Nat Neurosci. 2016;19:223-32.

23. Amaral DG, Schumann CM, Nordahl CW. Neuroanatomy of autism. Trends Neurosci. 2008;31:137-45.

24. Stoner R, Chow ML, Boyle MP, Sunkin SM, Mouton PR, Roy S, Wynshaw-Boris A, Colamarino SA, Lein ES, Courchesne E. Patches of disorganization in the neocortex of children with autism. N Engl J Med. 2014;370:1209-19.

25. Koch P, Opitz T, Steinbeck JA, Ladewig J, Brustle O. A rosette-type, selfrenewing human ES cell-derived neural stem cell with potential for in vitro instruction and synaptic integration. Proc Natl Acad Sci U S A. 2009;106: 3225-30.

26. Kang HJ, Kawasawa Yl, Cheng F, Zhu Y, Xu X, Li M, Sousa AM, Pletikos M, Meyer KA, Sedmak G, et al. Spatio-temporal transcriptome of the human brain. Nature. 2011;478:483-9.

27. Voineagu I, Wang X, Johnston P, Lowe JK, Tian Y, Horvath S, Mill J, Cantor RM, Blencowe BJ, Geschwind DH. Transcriptomic analysis of autistic brain reveals convergent molecular pathology. Nature. 2011;474:380-4.

28. Tasic B, Menon V, Nguyen TN, Kim TK, Jarsky T, Yao Z, Levi B, Gray LT, Sorensen SA, Dolbeare T, et al. Adult mouse cortical cell taxonomy revealed by single cell transcriptomics. Nat Neurosci. 2016;19:335-46.

29. Pasca SP, Portmann T, Voineagu I, Yazawa M, Shcheglovitov A, Pasca AM, Cord B, Palmer TD, Chikahisa S, Nishino S, et al. Using iPSC-derived neurons to uncover cellular phenotypes associated with Timothy syndrome. Nat Med. 2011;17:1657-62.

30. Li YL, Wang J, Zhang CY, Shen YQ, Wang HM, Ding L, Gu YC, Lou JT, Zhao $X T$, Ma ZL, Jin YX. MiR-146a-5p inhibits cell proliferation and cell cycle progression in NSCLC cell lines by targeting CCND1 and CCND2. Oncotarget. 2016;7:59287-98

31. Lowell S, Benchoua A, Heavey B, Smith AG. Notch promotes neural lineage entry by pluripotent embryonic stem cells. PLoS Biol. 2006;4:e121.

32. Sanchez-Mendoza EH, Bellver-Landete V, Arce C, Doeppner TR, Hermann DM, Oset-Gasque MJ. Vesicular glutamate transporters play a role in neuronal differentiation of cultured SVZ-derived neural precursor cells. PLoS One. 2017;12:e0177069

33. Hennchen M, Stubbusch J Abarchan-El Makhfi I, Kramer M, Deller T, PierreEugene C, Janoueix-Lerosey I, Delattre O, Ernsberger U, Schulte JB, Rohrer H.
Lin28B and Let-7 in the control of sympathetic neurogenesis and neuroblastoma development. J Neurosci. 2015;35:16531-44.

34. Hindley C, Philpott A. Co-ordination of cell cycle and differentiation in the developing nervous system. Biochem J. 2012:444:375-82.

35. Kwan KY, Sestan N, Anton ES. Transcriptional co-regulation of neuronal migration and laminar identity in the neocortex. Development. 2012;139:1535-46.

36. Courchesne E, Mouton PR, Calhoun ME, Semendeferi K, Ahrens-Barbeau C, Hallet MJ, Barnes CC, Pierce K. Neuron number and size in prefrontal cortex of children with autism. JAMA. 2011;306:2001-10.

37. Hutsler JJ, Zhang H. Increased dendritic spine densities on cortical projection neurons in autism spectrum disorders. Brain Res. 2010;1309:83-94.

38. Fang WQ, Chen WW, Jiang L, Liu K, Yung WH, Fu AK, Ip NY. Overproduction of upper-layer neurons in the neocortex leads to autism-like features in mice. Cell Rep. 2014;9:1635-43.

39. Wegiel J, Kuchna I, Nowicki K, Imaki H, Wegiel J, Marchi E, Ma SY, Chauhan A, Chauhan V, Bobrowicz TW, et al. The neuropathology of autism: defects of neurogenesis and neuronal migration, and dysplastic changes. Acta Neuropathol. 2010;119:755-70.

40. Hashemi E, Ariza J, Rogers H, Noctor SC, Martinez-Cerdeno V. The number of parvalbumin-expressing interneurons is decreased in the medial prefrontal cortex in autism. Cereb Cortex. 2017;27:1931-43.

41. Zoghbi HY, Bear MF. Synaptic dysfunction in neurodevelopmental disorders associated with autism and intellectual disabilities. Cold Spring Harb Perspect Biol. 2012;4. https://doi.org/10.1101/cshperspect.a009886.

42. Courchesne E, Pierce K, Schumann CM, Redcay E, Buckwalter JA, Kennedy DP, Morgan J. Mapping early brain development in autism. Neuron. 2007;56: 399-413.

43. Vaccarino FM, Smith KM. Increased brain size in autism - what it will take to solve a mystery. Biol Psychiatry. 2009;66:313-5.

44. Casanova EL, Casanova MF. Genetics studies indicate that neural induction and early neuronal maturation are disturbed in autism. Front Cell Neurosci. 2014;8:397.

45. Casanova MF. Autism as a sequence: from heterochronic germinal cell divisions to abnormalities of cell migration and cortical dysplasias. Med Hypotheses. 2014;83:32-8.

\section{Ready to submit your research? Choose BMC and benefit from:}

- fast, convenient online submission

- thorough peer review by experienced researchers in your field

- rapid publication on acceptance

- support for research data, including large and complex data types

- gold Open Access which fosters wider collaboration and increased citations

- maximum visibility for your research: over $100 \mathrm{M}$ website views per year

At BMC, research is always in progress.

Learn more biomedcentral.com/submissions 